\title{
Association between Hemoglobin A1c and Stroke Risk in Patients with Type 2 Diabetes
}

\author{
Yun Shen, ${ }^{\mathrm{a}}$ Lizheng Shi, ${ }^{\mathrm{b}}$ Elizabeth Nauman, ${ }^{\mathrm{c}}$ Peter Katzmarzyk, ${ }^{\mathrm{a}}$ Eboni Price-Haywood, ${ }^{\mathrm{d}}$ \\ Alessandra Bazzano, ${ }^{\mathrm{b}}$ Somesh Nigam, ${ }^{\mathrm{e}}$ Gang $\mathrm{Hu}^{\mathrm{a}}$ \\ aPennington Biomedical Research Center, Baton Rouge, LA, USA \\ ${ }^{b}$ Tulane University School of Public Health and Tropical Medicine, New Orleans, LA, USA \\ 'Louisiana Public Health Institute, New Orleans, LA, USA \\ ${ }^{\mathrm{d} O}$ Ochsner Health System Center for Outcomes and Health Services Research, New Orleans, LA, USA \\ 'Blue Cross and Blue Shield of Louisiana, Baton Rouge, LA, USA
}

Background and Purpose The association between hemoglobin A1c ( $\mathrm{HbA} 1 \mathrm{c})$ and stroke risk along with its subtypes is rarely reported. We aimed to investigate the association between $\mathrm{HbA1c}$ and the risk of incident stroke in patients with type 2 diabetes based on real world data from three healthcare systems.

Methods We performed a retrospective cohort study of 27,113 African Americans and 40,431 whites with type 2 diabetes. Demographic, anthropometric, laboratory, and medication information were abstracted from the National Patient-Centered Clinical Research Network common data model. Incident stroke events including both ischemic and hemorrhagic stroke were defined.

Results During a mean follow-up period of $3.79 \pm 1.68$ years, 7,735 patients developed stroke $(6,862$ ischemic and 873 hemorrhagic). Multivariable-adjusted hazard ratios across levels of HbA1c at baseline $(<6.0 \%, 6.0 \%$ to $6.9 \%$ [reference group], $7.0 \%$ to $7.9 \%, 8.0 \%$ to $8.9 \%, 9.0 \%$ to $9.9 \%$, and $\geq 10 \%)$ were $1.07,1.00,1.13,1.23,1.27$, and $1.37\left(P_{\text {trend }}<0.001\right)$ for total stroke, $1.02,1.00,1.13$, $1.20,1.24$, and $1.35\left(P_{\text {trend }}<0.001\right)$ for ischemic stroke, and $1.40,1.00,1.14,1.47,1.47$, and 1.51 $\left(P_{\text {trend }}=0.002\right)$ for hemorrhagic stroke. When we used an updated mean value of HbA1c, the $\mathrm{U}$-shaped association of $\mathrm{HbA} 1 \mathrm{c}$ with stroke risk did not change. This U-shaped association was consistent among patients of different subgroups. The U-shaped association was more pronounced among patients taking antidiabetic, lipid-lowering, and antihypertensive medications compared with those without these medications.

Conclusions These data suggest that diabetes management may have to be individualized according to the guideline recommendations rather than intensively attempting to lower $\mathrm{HbA1c}$.
Correspondence: Gang Hu Pennington Biomedical Research Center, 6400 Perkins Road, Baton Rouge, LA 70808, USA

Tel: $+1-2257633053$

Fax: +1-2257633009

E-mail: gang.hu@pbrc.edu

Received: July 2, 2019

Revised: October 10, 2019

Accepted: October 10, 2019

Keywords Glycated hemoglobin A; Stroke; Real world 


\section{Introduction}

Type 2 diabetes has emerged as a pandemic globally, with very high rates in the United States. For example, estimated cases of diabetes increased by 9 million in the United States between 2003-2004 and 2013-2014, affecting 30.2 million (13\%) adults in 2013-2014. ' Cardiovascular disease (CVD) is the leading cause of death among patients with diabetes, including heart disease and stroke. According to data from 2014, a total of 251,000 hospital discharges were reported with diabetes and stroke among United States adults. ${ }^{2}$ Poor glycemic control is significantly associated with diabetes comorbidities and mortality ${ }_{i}{ }^{2}$ therefore, an individual glycemic goal is recommended by many study groups or diabetes associations to delay the onset of diabetic complications. Hemoglobin A1C (HbA1c), as a good indicator of glycemic level over 2 or 3 months, is commonly used in clinical practice. While a high $\mathrm{HbA1c}$ level has been associated with a higher risk of CVD, some studies have suggested that a low $\mathrm{HbA1c}$ level may also be associated with a higher risk of CVD. ${ }^{4}$ The relationship between $\mathrm{HbA} 1 \mathrm{c}$ and CVD hospitalizations and all-cause mortality has appeared to be U-shaped among patients with type 2 diabetes. ${ }^{5}$ However, the association between $\mathrm{HbA} 1 \mathrm{c}$ and stroke risk along with its subtypes is rarely reported. Therefore, we aimed to investigate the association between $\mathrm{HbA} 1 \mathrm{c}$ and the risk of total stroke and its subtypes in patients with type 2 diabetes based on analyses of electronic medical record (EMR) data from three healthcare systems.

\section{Methods}

\section{Study participants}

Data on patients with type 2 diabetes in the Louisiana Experiment Assessing Diabetes outcomes (LEAD) cohort study were obtained through the Research Action for Health Network (REACHnet). ${ }^{6,7}$ REACHnet is a partnership of health systems, academic centers, and public health organizations that constitute an innovative data network for conducting efficient, multi-site research. Data from three REACHnet partner health systems were used in the current study including Ochsner Health System, Tulane Medical Center, and Access Health Louisiana. All patients' data were extracted from EMRs in these three health systems. The dataset included electronic health record data for the study cohort between January 1, 2013 and July 31,2018 . A unique global identifier was used to link records across the three health systems to avoid duplication of individual patients in the pooled dataset. In total, 18,706 patients (out of 203,701 records) were identified as duplicates across the three partner health systems.

The definition of type 2 diabetes in the present study was formulated according to the Surveillance, PREvention, and Management of Diabetes Mellitus (SUPREME-DM) criteria ${ }^{8}$ as follows: (1) one or more of the International Classification of Disease, Ninth Revision, Clinical Modification (ICD-9-CM) codes and Tenth Revision, Clinical Modification (ICD-10-CM) codes for type 2 diabetes associated with in-patient encounters; (2) two or more ICD codes associated with out-patient encounters on different days within 2 years; (3) combination of two or more of the following associated with out-patient encounters on different days within 2 years: 1) ICD codes; 2) fasting glucose level $\geq 126 \mathrm{mg} / \mathrm{dL}$; 3) 2-hour glucose level $\geq 200 \mathrm{mg} / \mathrm{dL}$; 4) random glucose $\geq 200 \mathrm{mg} / \mathrm{dL}$; 5) HbA1c $\geq 6.5 \%$; and 6) prescription for an antidiabetic medication. A total of 107,562 patients between the ages of 30 and 94 years were identified. After the exclusion of patients with prior CVDs and incomplete data, the present study included 67,544 patients with diabetes $(40,431$ whites and 27,113 African Americans). Compared with patients with diabetes excluded from the present study, the patients included had similar ages $(66.5 \pm 12.1$ years of age vs. $66.3 \pm 12.5$ years of age) with more African Americans (40.1\% vs. $36.2 \%$ ) and slightly fewer men (47.5\% vs. $49.1 \%$ ).

Standard protocol approvals, registrations, and patient consents

The study and analysis plan were approved by the Pennington Biomedical Research Center, Tulane University, and Ochsner Health System Institutional Review Boards. Registrations of this study at clinicaltrials.gov (https://clinicaltrials.gov) or somewhere else were not applicable. We used an electronic dataset compiled from medical records but not containing personally identifiable information except for the date of birth; thus, we did not obtain written informed consent from patients in the study cohort.

\section{Baseline measurements}

The National Patient-Centered Clinical Research Network (PCORnet) common data model is a specification that defines a standard organization and representation of data for the PCORnet distributed research network. ${ }^{9}$ Patients' data extracted from this common data model for the present study included date of birth, race/ethnicity, sex, encounter dates, weight, height, body mass index (BMI), blood pressure, tobacco use, diagnoses of diabetes, atrial fibrillation and stroke and dates of diagnoses, laboratory test dates, total cholesterol, triglycerides, high-density lipoprotein cholesterol (HDL-C), low-density lipoprotein cholesterol (LDL-C), HbA1c, estimated glomerular filtration rate 
(eGFR), and medication prescriptions such as antihypertensive drugs, glucose-lowering drugs, lipid-lowering drugs, and antiplatelet or anticoagulant drugs. These data elements were collected starting from the date of diabetes diagnosis (baseline). Using smoking status reported at each clinical visit, we classified the patients into three groups: current smokers, ever smokers, and never smokers. The eGFR was estimated using the Modification of Diet in Renal Disease (MDRD). ${ }^{10}$

\section{Follow-up}

We created the follow-up database in electronic form by using the number assigned to each patient who visited the health system with a unique patient identifier. The updated mean value of $\mathrm{HbA1c}$ was calculated for each participant from baseline to each year of follow-up. For example, after 1 year, the updated mean was the average of the baseline and 1-year values, and after 3 years it was the average of baseline, 1-, 2-, and 3 -year values. In the case of an event occurring during followup, the period for estimating the updated mean value was from baseline to the year before the event occurred. The average number of $\mathrm{HbA} 1 \mathrm{c}$ measurements during the follow-up period was 6.64. Stroke (ischemic or hemorrhagic) was the primary outcome in the present analysis. ICD-9-CM and ICD-10-CM codes were used to identify hemorrhagic stroke (ICD-9-CM codes 430-432 and ICD-10-CM codes I60-I62), ischemic stroke (ICD-9-CM codes 433-436 and ICD-10-CM codes I63I64), and any stroke (ICD-9-CM codes 430-436 and ICD-10CM codes I60-I64) events. The distributions of all ICD-9 and ICD-10 codes were: 430 (1.8\%), 431 (3.4\%), 432 (1.7\%), 433 (39.4\%), 434 (23.1\%), 435 (5.5\%), 436 (0.6\%), I60 (1.9\%), I61 (2.4\%), I62 (1.5\%), and I63 (18.7\%). These diagnoses were recorded in the course of routine patient care by the patients' treating clinicians. Incident stroke events were defined only when they occurred after the first record of diabetes diagnosis. The duration of follow-up for each cohort member (personyears) was tabulated from the date of the first documented diabetes diagnosis to the date of diagnosis of the outcome, death of inpatients or July 31, 2018. Either the admission date, the visiting date of emergency room or the visiting date of outpatient clinic was used when the ICD codes for stroke were first recorded. Diagnosis of stroke events could be made in either outpatient, inpatient or emergency encounters. Encounter types including ambulatory visit and other ambulatory visit were considered as outpatient encounters, while encounter types including inpatient, emergency department, emergency admission to inpatient, institutional stay, observation stay and institutional consult were considered as either inpatient or emergency encounters.

\section{Statistical analyses}

Cox proportional hazards regression was used to estimate hazard ratios (HRs) for incident stroke according to levels of $\mathrm{HbA1c}$. HbA1c was evaluated in the following two ways: (1) as categories $(<6.0 \%, 6.0 \%$ to $6.9 \%$ [reference group], $7.0 \%$ to $7.9 \%, 8.0 \%$ to $8.9 \%, 9.0 \%$ to $9.9 \%$, and $\geq 10 \%$ ); and (2) as a continuous variable when restricted cubic spline curves were performed. HbA1c levels were included in the models as dummy variables, and the significance of the trend across categories of $\mathrm{HbA1c}$ was tested in the same models by giving an ordinal numeric value for each dummy variable. The proportional hazards assumption in the Cox model was assessed with graphical methods and with models including time-by-covariate interactions. In general, all proportionality assumptions were appropriate. All analyses were first carried out adjusting for age and sex, and further for race, BMI, systolic blood pressure, LDL-C, HDL-C, triglycerides, eGFR, smoking, insurance type, use of antihypertensive drugs, use of glucose lowering drugs, use of lipid-lowering drugs, use of antiplatelet or anticoagulant drugs, and presence of atrial fibrillation. Sensitivity analyses were performed among patients who were diagnosed with stroke only in inpatient or emergency encounters. Statistical significance was considered to be $P<0.05$. $P$ for trend indicated the $P$-value for an overall linear trend. All statistical analyses were performed by using IBM SPSS Statistics for Windows version 24.0 (IBM Corp., Armonk, NY, USA) and SAS for Windows version 9.3 (SAS Institute Inc., Cary, NC, USA).

\section{Data availability statement}

The data that support the findings of this study are available from REACHnet but restrictions apply to the availability of these data, which were used under license for the current study, and so are not publicly available. Data are however available from the authors upon reasonable request and with permission of REACHnet.

\section{Results}

The baseline characteristics of patients in all categories of baseline $\mathrm{HbA1c}$ level are presented in Table 1. As the baseline $\mathrm{HbA1c}$ level increased, the values of BMI, systolic and diastolic blood pressure, total and LDL-C, and the proportion of male, African American ethnicity, current smokers, and glucose lowering medication users tended to increase, whereas the mean age and the proportion of patients taking antiplatelets or anticoagulants, atrial fibrillation, and medicare insurance coverage tended to decrease. The frequency of patients taking lipid-lowering drugs and antihypertensive agents showed an overall de- 
Table 1. Baseline characteristics of patients with type 2 diabetes by baseline hemoglobin A1c level

\begin{tabular}{|c|c|c|c|c|c|c|c|}
\hline \multirow{2}{*}{ Characteristic } & \multicolumn{6}{|c|}{ Hemoglobin A1c (\%) } & \multirow{2}{*}{$P$ for trend } \\
\hline & $<6.0$ & $6.0-6.9$ & $7.0-7.9$ & $8.0-8.9$ & $9.0-9.9$ & $\geq 10$ & \\
\hline No. of participants & 11,487 & 25,160 & 12,934 & 6,516 & 3,839 & 7,608 & \\
\hline Age (yr) & $67.4 \pm 12.6$ & $68.2 \pm 12.0$ & $67.6 \pm 11.7$ & $65.7 \pm 11.5$ & $63.2 \pm 11.3$ & $60.0 \pm 10.5$ & $<0.001$ \\
\hline Male sex (\%) & 45.9 & 45.2 & 48.2 & 49.8 & 52.2 & 52.0 & $<0.001$ \\
\hline Race (\%) & & & & & & & $<0.001$ \\
\hline African American & 34.4 & 39.0 & 38.7 & 40.5 & 44.6 & 52.5 & \\
\hline White & 65.6 & 61.0 & 61.3 & 59.5 & 55.4 & 47.5 & \\
\hline Body mass index $\left(\mathrm{kg} / \mathrm{m}^{2}\right)$ & $31.4 \pm 7.7$ & $32.8 \pm 7.5$ & $33.2 \pm 7.3$ & $33.5 \pm 7.5$ & $33.7 \pm 7.4$ & $33.2 \pm 7.4$ & $<0.001$ \\
\hline Body mass index categories (\%) & & & & & & & $<0.001$ \\
\hline$<25 \mathrm{~kg} / \mathrm{m}^{2}$ & 19.6 & 11.9 & 10.1 & 9.1 & 8.9 & 10.9 & \\
\hline $25-29.9 \mathrm{~kg} / \mathrm{m}^{2}$ & 29.0 & 28.0 & 26.8 & 26.2 & 25.4 & 25.3 & \\
\hline $30.0-34.9 \mathrm{~kg} / \mathrm{m}^{2}$ & 25.3 & 27.9 & 29.3 & 28.3 & 27.6 & 28.4 & \\
\hline$\geq 35 \mathrm{~kg} / \mathrm{m}^{2}$ & 26.2 & 32.2 & 33.9 & 36.4 & 38.1 & 35.4 & \\
\hline \multicolumn{8}{|l|}{ Blood pressure $(\mathrm{mm} \mathrm{Hg})$} \\
\hline Systolic & $132 \pm 14$ & $133 \pm 13$ & $134 \pm 13$ & $135 \pm 13$ & $135 \pm 13$ & $135 \pm 14$ & $<0.001$ \\
\hline Diastolic & $74 \pm 9$ & $75 \pm 8$ & $75 \pm 8$ & $76 \pm 8$ & $77 \pm 8$ & $78 \pm 8$ & $<0.001$ \\
\hline Total cholesterol (mg/dL) & $167 \pm 41.0$ & $170 \pm 37.2$ & $167 \pm 37.8$ & $169 \pm 40.0$ & $173 \pm 41.4$ & $183 \pm 46.0$ & $<0.001$ \\
\hline $\mathrm{LDL}-\mathrm{C}(\mathrm{mg} / \mathrm{dL})$ & $95.7 \pm 32.4$ & $98.1 \pm 30.8$ & $95.1 \pm 31.5$ & $96.4 \pm 32.6$ & $99.6 \pm 34.1$ & $108 \pm 37.6$ & $<0.001$ \\
\hline $\mathrm{HDL}-\mathrm{C}(\mathrm{mg} / \mathrm{dL})$ & $46.0 \pm 14.5$ & $45.2 \pm 12.2$ & $43.3 \pm 11.7$ & $42.3 \pm 11.8$ & $42.1 \pm 11.8$ & $42.2 \pm 12.1$ & $<0.001$ \\
\hline Triglycerides (mg/dL) & $129 \pm 77.3$ & $136 \pm 70.7$ & $148 \pm 81.9$ & $156 \pm 93.3$ & $164 \pm 133$ & $173 \pm 122$ & $<0.001$ \\
\hline Estimated GFR (\%) & & & & & & & $<0.001$ \\
\hline$\geq 90 \mathrm{~mL} / \mathrm{min} / 1.73 \mathrm{~m}^{2}$ & 14.7 & 13.8 & 13.4 & 12.6 & 14.6 & 16.1 & \\
\hline $60-89 \mathrm{~mL} / \mathrm{min} / 1.73 \mathrm{~m}^{2}$ & 51.6 & 58.7 & 56.5 & 55.8 & 55.4 & 55.2 & \\
\hline $30-59 \mathrm{~mL} / \mathrm{min} / 1.73 \mathrm{~m}^{2}$ & 23.0 & 23.3 & 25.2 & 25.9 & 25.0 & 24.5 & \\
\hline $15-29 \mathrm{~mL} / \mathrm{min} / 1.73 \mathrm{~m}^{2}$ & 4.5 & 2.4 & 3.2 & 3.2 & 3.1 & 2.9 & \\
\hline$<15 \mathrm{~mL} / \mathrm{min} / 1.73 \mathrm{~m}^{2}$ & 6.3 & 1.7 & 1.7 & 2.4 & 2.0 & 1.3 & \\
\hline Current smoker (\%) & 10.0 & 8.5 & 8.2 & 8.6 & 9.7 & 10.2 & $<0.001$ \\
\hline Insurance type (\%) & & & & & & & $<0.001$ \\
\hline Commercial/private & 31.2 & 35.6 & 36.8 & 38.4 & 42.2 & 47.8 & \\
\hline Medicare & 59.6 & 57.1 & 55.6 & 52.5 & 45.5 & 35.2 & \\
\hline Medicaid & 5.9 & 4.3 & 4.2 & 5.1 & 7.5 & 10.1 & \\
\hline Self-pay & 1.5 & 1.6 & 1.7 & 2.2 & 2.9 & 4.7 & \\
\hline Others & 1.8 & 1.4 & 1.7 & 1.8 & 1.9 & 2.2 & \\
\hline \multicolumn{8}{|l|}{ Use of medications (\%) } \\
\hline Lipid-lowering & 49.5 & 63.4 & 64.5 & 64.1 & 62.6 & 60.2 & $<0.001$ \\
\hline Antihypertensive & 74.8 & 78.4 & 78.9 & 78.9 & 77.1 & 74.2 & $<0.001$ \\
\hline Glucose-lowering & 49.9 & 65.6 & 82.5 & 85.9 & 87.0 & 90.5 & $<0.001$ \\
\hline Antiplatelet or anticoagulant & 42.7 & 42.3 & 41.1 & 40.3 & 39.3 & 37.2 & $<0.001$ \\
\hline Atrial fibrillation & 19.7 & 14.1 & 13.0 & 12.3 & 10.8 & 8.3 & $<0.001$ \\
\hline
\end{tabular}

Values are presented as mean \pm standard deviation.

LDL-C, low-density lipoprotein cholesterol; HDL-C, high-density lipoprotein cholesterol; GFR, glomerular filtration rate.

creasing trend as the baseline $\mathrm{HbA} 1 \mathrm{c}$ level increased except for the lowest HbA1c level category.

During a mean follow-up period of $3.79 \pm 1.68$ years, 7,735 patients developed stroke (6,862 ischemic and 873 hemorrhagic). A total of 2,604 inpatient deaths occurred including 554 cardiovascular deaths during the follow-up visits. Multivari- 
Table 2. Risk of total stroke, ischemic stroke and hemorrhagic stroke by baseline and mean follow-up hemoglobin A1c level

\begin{tabular}{|c|c|c|c|c|c|c|c|}
\hline \multirow{2}{*}{ Variable } & \multicolumn{6}{|c|}{ Hemoglobin A1c (\%) } & \multirow{2}{*}{$P$ for treno } \\
\hline & $<6.0$ & $6.0-6.9$ & $7.0-7.9$ & $8.0-8.9$ & $9.0-9.9$ & $\geq 10$ & \\
\hline \multicolumn{8}{|l|}{ Baseline hemoglobin A1c level } \\
\hline No. of patients & 11,487 & 25,160 & 12,934 & 6,516 & 3,839 & 7,608 & \\
\hline \multicolumn{8}{|l|}{ Total stroke } \\
\hline No. of cases & 1,316 & 2,702 & 1,607 & 830 & 458 & 822 & \\
\hline Person-years & 39,331 & 96,393 & 51,510 & 25,284 & 14,618 & 27,575 & \\
\hline Age- and sex-adjustment & $1.12(1.05-1.21)$ & 1.00 & $1.21(1.14-1.29)$ & $1.39(1.28-1.51)$ & $1.42(1.28-1.58)$ & $1.55(1.42-1.69)$ & $<0.001$ \\
\hline Multivariable adjustment & $1.07(1.01-1.14)$ & 1.00 & $1.13(1.06-1.21)$ & $1.23(1.13-1.33)$ & $1.27(1.14-1.40)$ & $1.37(1.26-1.49)$ & $<0.001$ \\
\hline \multicolumn{8}{|l|}{ Ischemic stroke } \\
\hline No. of cases & 1,103 & 2,420 & 1,451 & 736 & 410 & 742 & \\
\hline Person-years & 39,042 & 95,856 & 51,217 & 25,100 & 14,530 & 27,437 & \\
\hline Age-and sex-adjustment & $1.08(1.01-1.17)$ & 1.00 & $1.22(1.14-1.30)$ & $1.39(1.28-1.51)$ & $1.43(1.28-1.59)$ & $1.55(1.42-1.70)$ & $<0.001$ \\
\hline Multivariable adjustment & $1.02(1.01-1.05)$ & 1.00 & $1.13(1.06-1.21)$ & $1.20(1.10-1.31)$ & $1.24(1.12-1.39)$ & $1.35(1.24-1.48)$ & $<0.001$ \\
\hline \multicolumn{8}{|l|}{ Hemorrhagic stroke } \\
\hline No. of cases & 213 & 282 & 156 & 94 & 48 & 80 & \\
\hline Person-years & 37,957 & 93,207 & 49,537 & 24,299 & 14,034 & 36,469 & \\
\hline Age-and sex-adjustment & $1.65(1.31-2.07)$ & 1.00 & $1.10(0.90-1.33)$ & $1.49(1.18-1.88)$ & $1.50(1.10-2.04)$ & $1.60(1.24-2.07)$ & $<0.001$ \\
\hline Multivariable adjustment & $1.40(1.16-1.69)$ & 1.00 & $1.14(0.93-1.39)$ & $1.47(1.16-1.87)$ & $1.47(1.07-2.01)$ & $1.51(1.16-1.98)$ & 0.002 \\
\hline \multicolumn{8}{|c|}{ Mean follow-up hemoglobin A1c level } \\
\hline No. of patients & 8,630 & 22,172 & 22,977 & 6,808 & 3,491 & 3,466 & \\
\hline \multicolumn{8}{|l|}{ Total stroke } \\
\hline No. of cases & 1,169 & 2,431 & 2,383 & 887 & 444 & 421 & \\
\hline Person-years & 31,222 & 86,500 & 82,936 & 27,132 & 13,772 & 13,149 & \\
\hline Age- and sex-adjustment & $1.24(1.15-1.33)$ & 1.00 & $1.04(0.98-1.11)$ & $1.47(1.36-1.59)$ & $1.62(1.46-1.80)$ & $1.76(1.57-1.96)$ & $<0.001$ \\
\hline Multivariable adjustment & $1.18(1.10-1.27)$ & 1.00 & $1.07(1.01-1.13)$ & $1.25(1.16-1.35)$ & $1.42(1.28-1.58)$ & $1.52(1.36-1.69)$ & $<0.001$ \\
\hline \multicolumn{8}{|l|}{ Ischemic stroke } \\
\hline No. of cases & 983 & 2,191 & 2,107 & 806 & 402 & 373 & \\
\hline Person-years & 30,927 & 86,041 & 82,511 & 26,953 & 13,693 & 13,057 & \\
\hline Age-and sex-adjustment & $1.18(1.09-1.27)$ & 1.00 & $1.04(0.98-1.10)$ & $1.48(1.36-1.60)$ & $1.63(1.46-1.82)$ & $1.73(1.54-1.94)$ & $<0.001$ \\
\hline Multivariable adjustment & $1.12(1.04-1.21)$ & 1.00 & $1.05(0.99-1.12)$ & $1.24(1.14-1.35)$ & $1.40(1.26-1.56)$ & $1.46(1.30-1.64)$ & $<0.001$ \\
\hline \multicolumn{8}{|l|}{ Hemorrhagic stroke } \\
\hline No. of cases & 186 & 240 & 276 & 81 & 42 & 48 & \\
\hline Person-years & 29,929 & 83,506 & 80,367 & 25,964 & 13,141 & 12,595 & \\
\hline Age-and sex-adjustment & $2.04(1.61-2.59)$ & 1.00 & $1.12(0.90-1.40)$ & 1.39 (1.03-1.89) & $1.54(1.03-1.89)$ & $2.16(1.46-3.20)$ & 0.001 \\
\hline Multivariable adjustment & $1.67(1.36-2.04)$ & 1.00 & $1.19(1.00-1.42)$ & $1.33(1.03-1.72)$ & $1.60(1.14-2.24)$ & $2.07(1.50-2.87)$ & $<0.001$ \\
\hline
\end{tabular}

Values are presented as hazard ratio (95\% confidence interval) unless otherwise indicated. Multivariable adjustment included age, sex, race, body mass index, systolic blood pressure, low-density lipoprotein cholesterol, high-density lipoprotein cholesterol, triglycerides, estimated glomerular filtration rate, smoking, insurance type, use of antihypertensive drugs, use of glucose lowering drugs, use of lipid-lowering drugs, use of antiplatelet or anticoagulant drugs, and presence of atrial fibrillation.

able-adjusted HRs across levels of HbA1c at baseline $(<6.0 \%$, $6.0 \%$ to $6.9 \%$ [reference group], $7.0 \%$ to $7.9 \%, 8.0 \%$ to $8.9 \%$, $9.0 \%$ to $9.9 \%$, and $\geq 10 \%)$ were $1.07,1.00,1.13,1.23,1.27$, and $1.37\left(P_{\text {trend }}<0.001\right)$ for total stroke (Table 2$), 1.02,1.00,1.13$, $1.20,1.24$, and $1.35\left(P_{\text {trend }}<0.001\right)$ for ischemic stroke (Table 2$)$, and $1.40,1.00,1.14,1.47,1.47$, and $1.51\left(P_{\text {trend }}=0.002\right)$ for hemorrhagic stroke (Table 2). In analyses using the mean follow-up HbA1c level, the U-shaped association of $\mathrm{HbA1c}$ with the risk of total stroke, ischemic stroke, or hemorrhagic stroke remained unchanged (Table 2).

We performed two sensitivity analysis to confirm our findings. Using diagnoses associated with inpatient or emergency 

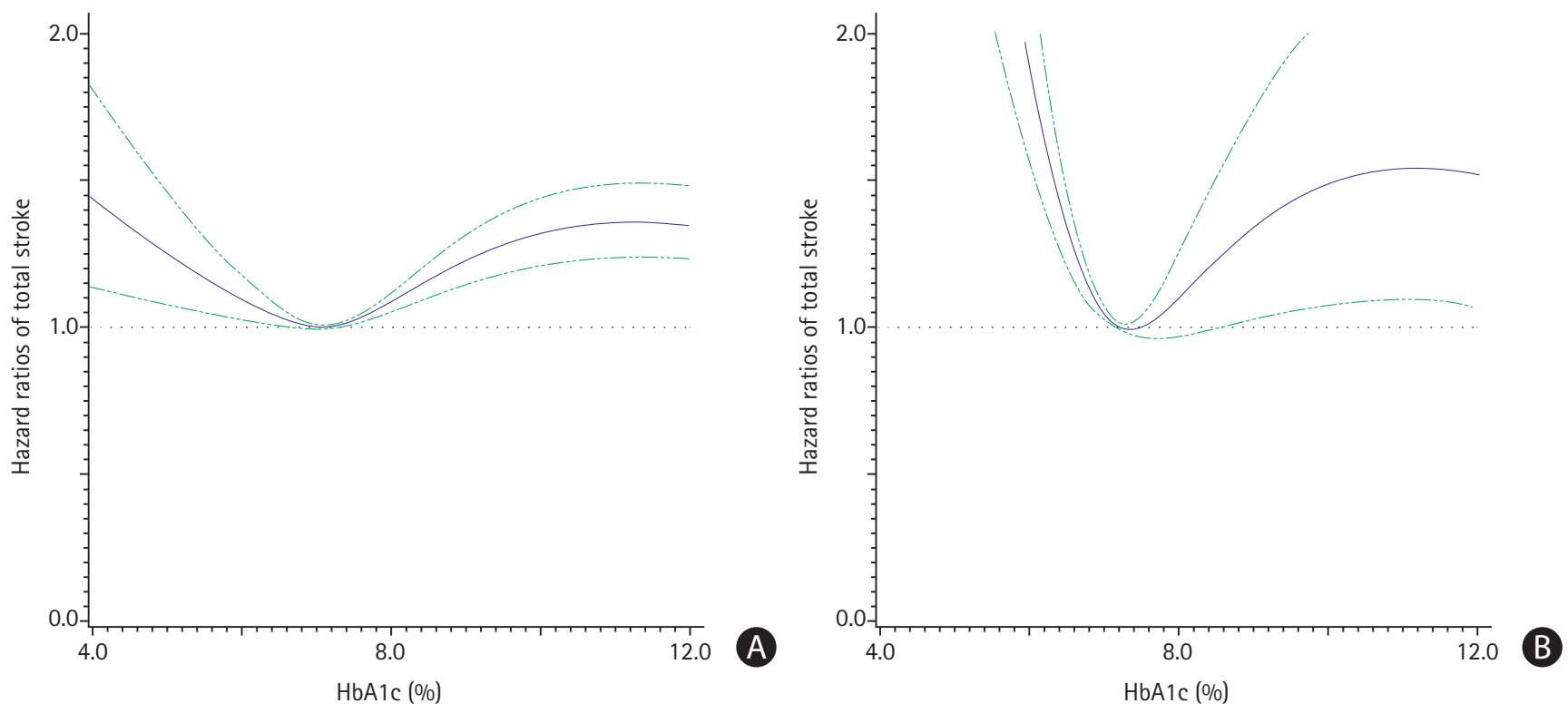

Figure 1. Hazard ratios of total stroke risks among patients with type 2 diabetes diagnosed in all encounters (A) and only in inpatients/emergency encounters (B) by different hemoglobin A1c (HbA1c) levels at baseline. Models were adjusted for age, sex, race, body mass index, systolic blood pressure, low-density lipoprotein cholesterol, high-density lipoprotein cholesterol, triglycerides, estimated glomerular filtration rate, smoking, insurance type, use of antihypertensive drugs, use of glucose lowering drugs, use of lipid-lowering drugs, use of antiplatelet or anticoagulant drugs, and presence of atrial fibrillation.

encounters was applied in the first sensitivity analysis. During a mean follow-up period of $3.95 \pm 1.58$ years, 2,946 patients developed stroke (2,623 ischemic and 323 hemorrhagic). Multivariable-adjusted HRs across levels of HbA1c at baseline $(<6.0 \%, 6.0 \%$ to $6.9 \%$ [reference group], $7.0 \%$ to $7.9 \%, 8.0 \%$ to $8.9 \%$, $9.0 \%$ to $9.9 \%$, and $\geq 10 \%)$ were $1.31,1.00,1.35,1.43$, 1.56 , and $1.72\left(P_{\text {trend }}<0.001\right)$ for total stroke (Supplementary Table 1), 1.16, 1.00, 1.30, 1.28, 1.59, and $1.91\left(P_{\text {trend }}<0.001\right)$ for ischemic stroke (Supplementary Table 1), and 1.93, 1.00, 1.59, $2.21,2.04$, and $1.89\left(P_{\text {trend }}<0.001\right)$ for hemorrhagic stroke (Supplementary Table 1). The U-shaped associations between both baseline and updated mean values of $\mathrm{HbA} 1 \mathrm{c}$ and the risk of incident stroke were observed with a greater slope in the sensitivity analysis. For the second sensitivity analysis, we excluded all deaths during the follow-up visits (Supplementary Table 2). A consistent $\mathrm{U}$-shaped association between $\mathrm{HbA1c}$ and stroke risks could also be found.

When $\mathrm{HbA} 1 \mathrm{c}$ level was considered as a continuous variable by using restricted cubic splines, a U-shaped association of $\mathrm{HbA1C}$ with the risk of stroke was observed (Figure 1A). The risk of stroke was lowest among patients with the baseline $\mathrm{HbA} 1 \mathrm{c}$ level of $6.0 \%$ to $7.0 \%$. When performing restricted cubic splines in the sensitivity analysis including patients who were diagnosed with stroke only in inpatient or emergency encounters, we found a similar but more pronounced U-shaped curve with a greater slope (Figure 1B).
When subgroup analyses were utilized (including sensitivity analysis), the U-shaped association between $\mathrm{HbA} 1 \mathrm{c}$ and the risk of total stroke was consistent among patients of different ages, races, sexes, BMI, never and past or current smokers (Table 3 and Supplementary Table 3). The U-shaped association was more pronounced among patients taking glucose lowering, lipid-lowering, antihypertensive, antiplatelet or anticoagulant drugs compared with those without these drugs.

\section{Discussion}

In this large health system-based cohort, we found that both lower $\mathrm{HbA} 1 \mathrm{c}$ and higher $\mathrm{HbA1c}$ levels were associated with higher risks of incident stroke, which formed a U-shaped curve. In the analysis restricted to stroke cases only diagnosed by inpatient or emergency, the U-shape association appears more pronounced. These results indicate that both intensive and poor glycemic control might be associated with an increased risk of stroke in patients with type 2 diabetes.

Diabetes is a leading cause of stroke, especially ischemic stroke. Patients with type 2 diabetes have an increased risk of stroke estimated to range from 150\% to 400\% compared with those without diabetes. ${ }^{11}$ It is common practice that $\mathrm{HbA} 1 \mathrm{c}$ is now widely used as a routine screening marker for glycemic control. Most current guidelines circulated by professional associations for diabetes including the American Diabetes Asso- 
Table 3. Subgroup analyses for the risk of total stroke by baseline hemoglobin A1c level

\begin{tabular}{|c|c|c|c|c|c|c|c|c|}
\hline \multirow{2}{*}{ Variable } & \multirow{2}{*}{$\begin{array}{c}\text { No. of } \\
\text { patients }\end{array}$} & \multicolumn{6}{|c|}{ Hemoglobin A1c (\%) } & \multirow{2}{*}{$P$ for trend } \\
\hline & & $<6.0$ & $6.0-6.9$ & $7.0-7.9$ & $8.0-8.9$ & $9.0-9.9$ & $\geq 10$ & \\
\hline \multicolumn{9}{|l|}{ Age (yr) } \\
\hline$<60$ & 20,512 & $1.01(0.82-1.24)$ & 1.00 & $1.05(0.86-1.29)$ & $1.16(0.92-1.46)$ & $1.36(1.07-1.73)$ & $1.43(1.19-1.72)$ & 0.001 \\
\hline$\geq 60$ & 47,032 & $1.02(0.95-1.10)$ & 1.00 & $1.12(1.05-1.20)$ & $1.18(1.09-1.29)$ & $1.15(1.03-1.29)$ & $1.18(1.07-1.30)$ & $<0.001$ \\
\hline \multicolumn{9}{|l|}{ Sex } \\
\hline Men & 32,073 & $1.02(0.88-1.08)$ & 1.00 & $1.14(1.04-1.24)$ & $1.17(1.04-1.32)$ & $1.14(0.99-1.33)$ & $1.35(1.19-1.53)$ & $<0.001$ \\
\hline Women & 35,471 & $1.15(1.05-1.26)$ & 1.00 & $1.12(1.02-1.22)$ & $1.27(1.14-1.41)$ & $1.38(1.20-1.59)$ & $1.37(1.22-1.54)$ & $<0.001$ \\
\hline \multicolumn{9}{|l|}{ Race } \\
\hline African Americans & 27,113 & $1.12(1.00-1.26)$ & 1.00 & $1.11(1.00-1.24)$ & $1.24(1.09-1.40)$ & $1.44(1.25-1.67)$ & $1.39(1.23-1.57)$ & $<0.001$ \\
\hline Whites & 40,431 & $1.04(0.95-1.13)$ & 1.00 & $1.14(1.06-1.24)$ & $1.23(1.11-1.36)$ & $1.13(0.98-1.31)$ & $1.35(1.20-1.52)$ & $<0.001$ \\
\hline \multicolumn{9}{|l|}{$\begin{array}{l}\text { Body mass index } \\
\left(\mathrm{kg} / \mathrm{m}^{2}\right)\end{array}$} \\
\hline$<30$ & 26,747 & $1.13(1.04-1.24)$ & 1.00 & $1.11(1.01-1.21)$ & $1.20(1.07-1.35)$ & $1.17(1.00-1.36)$ & $1.30(1.15-1.48)$ & $<0.001$ \\
\hline$\geq 30$ & 40,797 & $1.00(0.99-1.02)$ & 1.00 & $1.14(1.04-1.24)$ & $1.23(1.10-1.37)$ & $1.33(1.16-1.52)$ & $1.42(1.27-1.59)$ & $<0.001$ \\
\hline \multicolumn{9}{|l|}{ Smoking status } \\
\hline Never smoking & 53,959 & $1.04(0.96-1.13)$ & 1.00 & $1.05(0.91-1.20)$ & 1.16 (0.98-1.38) & $1.12(0.89-1.40)$ & $1.33(1.10-1.59)$ & 0.037 \\
\hline $\begin{array}{l}\text { Past and current } \\
\text { smoking }\end{array}$ & 13,585 & $1.14(0.99-1.31)$ & 1.00 & $1.17(1.09-1.25)$ & $1.26(1.15-1.38)$ & $1.33(1.18-1.49)$ & $1.39(1.26-1.53)$ & $<0.001$ \\
\hline \multicolumn{9}{|l|}{$\begin{array}{l}\text { Using glucose-low- } \\
\text { ering drugs* }\end{array}$} \\
\hline No & 18,810 & $1.08(0.97-1.20)$ & 1.00 & $1.09(0.93-1.28)$ & $1.16(0.92-1.46)$ & $1.12(0.81-1.56)$ & $1.22(0.91-1.65)$ & 0.451 \\
\hline Yes & 48,734 & $1.06(0.97-1.16)$ & 1.00 & $1.14(1.06-1.22)$ & $1.24(1.14-1.35)$ & $1.29(1.16-1.44)$ & $1.40(1.28-1.53)$ & $<0.001$ \\
\hline $\begin{array}{l}\text { Insulin or sulfo- } \\
\text { nylurea }\end{array}$ & 20,573 & $1.04(0.93-1.16)$ & 1.00 & $1.02(0.94-1.11)$ & $1.11(1.01-1.22)$ & $1.10(0.98-1.23)$ & $1.16(1.06-1.28)$ & 0.017 \\
\hline Others & 28,161 & $1.10(1.01-1.20)$ & 1.00 & $1.09(0.98-1.22)$ & $1.00(0.83-1.20)$ & $1.12(0.86-1.45)$ & $1.09(0.86-1.39)$ & 0.491 \\
\hline \multicolumn{9}{|l|}{$\begin{array}{l}\text { Using lipid-lowering } \\
\text { drugs* }^{*}\end{array}$} \\
\hline No & 26,396 & $1.04(0.92-1.18)$ & 1.00 & $1.21(1.06-1.38)$ & $1.18(1.00-1.40)$ & $1.31(1.07-1.62)$ & $1.28(1.08-1.52)$ & 0.005 \\
\hline Yes & 41,148 & $1.07(1.01-1.16)$ & 1.00 & $1.12(1.04-1.20)$ & $1.25(1.14-1.37)$ & $1.26(1.12-1.42)$ & $1.41(1.28-1.55)$ & $<0.001$ \\
\hline \multicolumn{9}{|l|}{$\begin{array}{l}\text { Using antihyperten- } \\
\text { sive drugs* }\end{array}$} \\
\hline No & 15,278 & $1.19(0.98-1.44)$ & 1.00 & $1.12(0.92-1.36)$ & $1.27(0.99-1.63)$ & $1.10(0.79-1.52)$ & $1.21(0.94-1.56)$ & 0.333 \\
\hline Yes & 52,266 & $1.05(0.97-1.13)$ & 1.00 & $1.13(1.06-1.21)$ & $1.22(1.12-1.33)$ & $1.28(1.15-1.43)$ & $1.39(1.27-1.52)$ & $<0.001$ \\
\hline \multicolumn{9}{|l|}{$\begin{array}{l}\text { Using antiplatelet or } \\
\text { anticoagulant } \\
\text { drugs* }\end{array}$} \\
\hline No & 52,949 & $1.08(0.99-1.18)$ & 1.00 & $1.13(1.04-1.23)$ & $1.31(1.19-1.45)$ & $1.33(1.16-1.52)$ & $1.36(1.22-1.53)$ & $<0.001$ \\
\hline Yes & 14,595 & $1.04(0.94-1.16)$ & 1.00 & $1.12(1.01-1.24)$ & $1.09(0.96-1.24)$ & $1.17(1.00-1.36)$ & $1.31(1.16-1.48)$ & 0.001 \\
\hline \multicolumn{9}{|l|}{$\begin{array}{l}\text { Presence of atrial } \\
\text { fibrillation* }\end{array}$} \\
\hline No & 58,200 & $1.13(1.04-1.23)$ & 1.00 & $1.13(1.05-1.22)$ & $1.27(1.16-1.40)$ & $1.27(1.12-1.42)$ & $1.40(1.27-1.54)$ & $<0.001$ \\
\hline Yes & 9,344 & $1.01(0.85-1.08)$ & 1.00 & $1.13(1.01-1.27)$ & $1.07(0.91-1.25)$ & $1.24(1.01-1.52)$ & $1.26(1.06-1.51)$ & 0.031 \\
\hline
\end{tabular}

Values are presented as hazard ratio (95\% confidence interval) unless otherwise indicated. All hazard ratios were adjusted for age, sex, race, body mass index, systolic blood pressure, low-density lipoprotein cholesterol, high-density lipoprotein cholesterol, triglycerides, estimated glomerular filtration rate, smoking, insurance type, use of antihypertensive drugs, use of glucose lowering drugs, use of lipid-lowering drugs, use of antiplatelet or anticoagulant drugs, and presence of atrial fibrillation other than the variable for stratification.

${ }^{*} P$ for interaction $<0.05$. 
Table 4. Summary of the findings of previous population-based studies since 2009

\begin{tabular}{|c|c|c|c|c|c|c|c|}
\hline Year & Country & Data source & $\begin{array}{l}\text { Sample } \\
\text { size }\end{array}$ & $\begin{array}{l}\text { No. of } \\
\text { cases }\end{array}$ & Age (yr) & $\begin{array}{l}\text { Mean or } \\
\text { median } \\
\text { follow-up }\end{array}$ & $\mathrm{HR}$ or $\mathrm{RR}(95 \% \mathrm{Cl})$ \\
\hline $\begin{array}{l}\text { Cederholm et al. } \\
(2009)^{23}\end{array}$ & Sweden & $\begin{array}{l}\text { Swedish National } \\
\text { Diabetes Register }\end{array}$ & 4,753 & 238 & $30-70$ & 5.7 years & $\begin{array}{l}\text { 7.5\%-9.0\%: reference } \\
<7.5 \% \text { : } 0.47 \text { (0.36-0.63) }\end{array}$ \\
\hline $\begin{array}{l}\text { Camafort et al. } \\
(2011)^{24}\end{array}$ & Spain & FRENA Registry & 974 & 29 & $68 \pm 9.5$ & 14 months & $\begin{array}{l}>7 \% \text { : reference } \\
<7 \% \text { : } 0.9(0.4-1.8)\end{array}$ \\
\hline $\begin{array}{l}\text { Skriver et al. } \\
(2012)^{13}\end{array}$ & Denmark & $\begin{array}{l}\text { Electronic medical } \\
\text { records }\end{array}$ & 11,747 & 287 & $\begin{array}{l}\mathrm{HbA1c}<7 \%: 67 \text { (57-77) } \\
\mathrm{HbA} 1 \mathrm{c} \geq 7 \%: 65 \text { (56-74) }\end{array}$ & 730 days & $\begin{array}{l}<7 \%: \text { reference } \\
\geq 7 \%: 1.00(0.78-1.27)\end{array}$ \\
\hline $\begin{array}{l}\text { Hayashi et al. } \\
(2013)^{25}\end{array}$ & Japan & JCDM cohort & 4,014 & 104 & $67.4 \pm 9.5$ years old & 5.5 years & Per 1\% increase: 1.171 (1.001-1.356) \\
\hline $\begin{array}{l}\text { Lin et al. } \\
\qquad(2014)^{21}\end{array}$ & $\begin{array}{r}\text { Taiwan, } \\
\text { China }\end{array}$ & NDCMP cohort & 63,084 & 2,250 & $\geq 30$ & 7.5 years & $\begin{array}{l}\text { <7.0\%: reference } \\
\text { 7.0\%-8.0\%: } 1.27(1.13-1.43) \\
\text { 8.0\%-9.0\%: } 1.55(1.37-1.75) \\
\geq 9.0 \%: 2.06(1.85-2.31)\end{array}$ \\
\hline $\begin{array}{l}\text { Zhao et al. } \\
\qquad(2014)^{22}\end{array}$ & USA & $\begin{array}{l}\text { Electronic medical } \\
\text { records }\end{array}$ & 30,154 & 2,949 & $\begin{array}{c}\text { Men: } 50.9 \pm 10.1 \\
\text { Women: } 51.5 \pm 10.1\end{array}$ & 6.7 years & $\begin{array}{l}\text { Men } \\
\text { <6.0\%: } 1.05 \text { (0.88-1.26) } \\
\text { 6.0\%-6.9\%: reference } \\
\text { 7.0\%-7.9\%: } 1.12 \text { (0.94-1.33) } \\
\text { 8.0\%-8.9\%: } 1.20(0.98-1.46) \\
\text { 9.0\%-9.9\%: } 1.23(0.98-1.54) \\
\geq 10.0 \%: 1.08 \text { (0.86-1.36) } \\
\text { Women } \\
\text { <6.0\%: } 1.06 \text { (0.93-1.21) } \\
\text { 6.0\%-6.9\%: reference } \\
\text { 7.0\%-7.9\%: } 1.11(0.97-1.27) \\
\text { 8.0\%-8.9\%: } 1.30(1.12-1.52) \\
\text { 9.0\%-9.9\%: } 1.41(1.19-1.68) \\
\geq 10.0 \%: 1.33(1.11-1.59)\end{array}$ \\
\hline $\begin{array}{l}\text { Kranenburg et } \\
\text { al. }(2015)^{20}\end{array}$ & $\begin{array}{l}\text { The Nether- } \\
\text { lands }\end{array}$ & SMART cohort & 1,687 & 62 & $60.2 \pm 10.2$ & 6.1 years & $\begin{array}{l}\text { Per 1\% increase: } \\
\text { Patients with vascular disease: } 1.03 \\
(0.81-1.31) \\
\text { Patients without vascular disease: } 1.40 \\
(1.01-1.94)\end{array}$ \\
\hline $\begin{array}{l}\text { Bots et al. } \\
(2016)^{14}\end{array}$ & $\begin{array}{l}\text { The Nether- } \\
\text { lands }\end{array}$ & SMART cohort & 1,096 & 48 & $62.6 \pm 8.8$ & 6.9 years & Per 1\% increase: 1.09 (0.84-1.41) \\
\hline 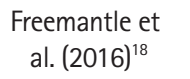 & International & CREDIT cohort & 2,999 & 57 & $>40$ & 54 months & Per 1\% increase: 1.363 (1.168-1.591) \\
\hline $\begin{array}{l}\text { Morita et al. } \\
(2017)^{15}\end{array}$ & Japan & $\begin{array}{l}\text { Community-dwell- } \\
\text { ing cohort }\end{array}$ & 184 & 7 & $65-94$ & 5 years & $\begin{array}{l}\geq 6.0 \% \text { : reference } \\
<6.0 \% \text { : non-significant }\end{array}$ \\
\hline $\begin{array}{l}\text { Hwang et al. } \\
(2017)^{16}\end{array}$ & Korea & $\begin{array}{l}\text { Electronic medical } \\
\text { records }\end{array}$ & 980 & 31 & $\begin{array}{l}\mathrm{HbA} 1 \mathrm{c}<7.0 \%: 62.9 \pm 10.0 \\
\mathrm{HbA} 1 \mathrm{c} \geq 7.0 \%: 62.7 \pm 10.1\end{array}$ & 717.6 days & $\begin{array}{l}<7.0 \% \text { : reference } \\
\geq 7.0 \%: 1.22(0.66-2.27)\end{array}$ \\
\hline $\begin{array}{l}\text { Heller et al. } \\
(2017)^{17}\end{array}$ & International & EXAMINE trial & 5,380 & 61 & 61.0 & 18 months & $\begin{array}{l}<7.0 \%: \text { reference } \\
\text { 7.0\%-8.0\%: } 1.73(0.81-3.71) \\
\text { 8.0\%-9.0\%: } 2.36(0.99-5.61) \\
\geq 9.0 \%: 1.68(0.56-5.01)\end{array}$ \\
\hline $\begin{array}{l}\text { Alatorre et al. } \\
(2018)^{26}\end{array}$ & UK & $\begin{array}{l}\text { Electronic medical } \\
\text { records }\end{array}$ & 82,151 & 2,127 & $\geq 18$ & 5 years & $\begin{array}{l}<6.0 \%: 1.29(1.02-1.63) \\
6.0 \%-6.49 \%: \text { NA } \\
6.5 \%-6.99 \%: \text { reference } \\
7.0 \%-7.49 \%: 1.49(1.24-1.79) \\
7.5 \%-8.0 \%: 4.67(2.92-7.45)\end{array}$ \\
\hline $\begin{array}{l}\text { Rawshani et al. } \\
(2018)^{27}\end{array}$ & Sweden & $\begin{array}{l}\text { Swedish National } \\
\text { Diabetes Register }\end{array}$ & 271,174 & 14,474 & $60.6 \pm 10.9$ & 5.7 years & $\begin{array}{l}\text { Levels below the guideline target levels } \\
\text { for glycated hemoglobin was associat- } \\
\text { ed with lower risks of stroke. }\end{array}$ \\
\hline $\begin{array}{l}\text { Fangel et al. } \\
(2019)^{19}\end{array}$ & Denmark & $\begin{array}{l}\text { Electronic medical } \\
\text { records }\end{array}$ & 5,386 & 253 & $72.7 \pm 9.7$ & 1.9 years & $\begin{array}{l}<48 \mathrm{mmol} / \mathrm{mol}: \text { reference } \\
49-58 \mathrm{mmol} / \mathrm{mol}: 1.49(1.09-2.05) \\
>58 \mathrm{mmol} / \mathrm{mol}: 1.59(1.13-2.22)\end{array}$ \\
\hline
\end{tabular}


Table 4. Continued

\begin{tabular}{|c|c|c|c|c|c|c|c|}
\hline Year & Country & Data source & $\begin{array}{l}\text { Sample } \\
\text { size }\end{array}$ & $\begin{array}{l}\text { No. of } \\
\text { cases }\end{array}$ & Age (yr) & $\begin{array}{l}\text { Mean or } \\
\text { median } \\
\text { follow-up }\end{array}$ & HR or $\mathrm{RR}(95 \% \mathrm{Cl})$ \\
\hline $\begin{array}{l}\text { Present study } \\
\text { (2019) }\end{array}$ & USA & $\begin{array}{l}\text { Electronic medical } \\
\text { records }\end{array}$ & 67,544 & 7,735 & $66.5 \pm 12.1$ & 3.8 years & $\begin{array}{l}<6.0 \%: 1.10(1.02-1.18) \\
\text { 6.0\%-6.9\%: reference } \\
\text { 7.0\%-7.9\%: } 1.16(1.08-1.23) \\
\text { 8.0\%-8.9\%: } 1.27(1.17-1.37) \\
9.0 \%-9.9 \%: 1.30(1.17-1.45) \\
\geq 10 \%: 1.40(1.28-1.53)\end{array}$ \\
\hline
\end{tabular}

$\mathrm{HR}$, hazard ratio; RR, relative risk; $\mathrm{Cl}$, confidence interval; FRENA, Factores de Riesgo y ENfermedad Arterial; HbA1c, hemoglobin A1c; JCDM, Japan Cholesterol and Diabetes Mellitus Study; NDCMP, National Diabetes Case Management Program; SMART, the Second Manifestations of ARTerial Disease; CREDIT, the Cardiovascular Risk Evaluation in people with Type 2 Diabetes on Insulin Therapy; EXAMINE, the Examination of Cardiovascular Outcomes with Alogliptin versus Standard of Care; NA, not applicable.

ciation $^{12}$ recommend $\mathrm{HbA} 1 \mathrm{C}<7 \%$ as the optimal target for glycemic control. Poor glycemic control would eventually contribute to a high risk of diabetic comorbidities such as stroke in patients with type 2 diabetes. However, studies with regard to this topic have shown inconsistent results. In the early 1990s, results from the United Kingdom Prospective Diabetes Study (UKPDS) ${ }^{3}$ showed that patients with $\mathrm{HbA1c}$ level less than $6.3 \%$ (reference) did not have a significantly lower risk of first-ever stroke than patients with $\mathrm{HbA} 1 \mathrm{c}$ level between $6.3 \%$ and $7.6 \%$ (odds ratio [OR], 1.2; 95\% confidence interval [Cl], 0.8 to 1.7$)$ and even those with $\mathrm{HbA} 1 \mathrm{c}$ level $>7.6 \%(\mathrm{OR}, 1.1$; $95 \% \mathrm{Cl}, 0.7$ to 1.6$)$. Another group using a large public health database also showed a non-significant association between $\mathrm{HbA} 1 \mathrm{c}$ and stroke risk ( $\mathrm{HR}, 1.00 ; 95 \% \mathrm{Cl}, 0.78$ to 1.27$).{ }^{13}$ Negative results regarding the association between $\mathrm{HbA} 1 \mathrm{c}$ and stroke were also reported by other researchers from different countries. ${ }^{14-17}$ In contrast, some national or international ${ }^{18}$ studies from Denmark, ${ }_{1}^{19}$ Netherlands, ${ }_{1}^{20}$ China (Taiwan), ${ }_{1}^{21}$ the United States, ${ }_{1}^{22}$ Sweden, ${ }_{1}^{23}$ Spain, ${ }_{1}^{24}$ and Japan ${ }^{25}$ showed a dose-response association between $\mathrm{HbA} 1 \mathrm{c}$ level and risk of first-ever stroke. Researches from 2009 to 2019 in terms of the association between $\mathrm{HbA} 1 \mathrm{c}$ and risk of stroke among patients with type 2 diabetes are summarized in Table 4 . In our previous study, ${ }_{1}^{22}$ we found a significant sex difference in the association between $\mathrm{HbA} 1 \mathrm{c}$ and stroke. There was a U-shaped association between $\mathrm{HbA} 1 \mathrm{c}$ and stroke in women but not in men, in which we used the same analytical method as applied in this analysis. However, the previous data source was totally different from the present one. The data from the Louisiana State University Health Care Services Division were used previously, which compromised over $75 \%$ poor patients without any type of insurance covered and over 50\% African Americans. Between-study differences in the population characteristics might account for the discrepant findings between the earlier and current studies.

However, it is not always true that lower $\mathrm{HbA1c}$ can result in a better quality of life or health outcomes, because some studies ${ }^{26,27}$ have indicated that extremely low $\mathrm{HbA} 1 \mathrm{c}$ may contrarily contribute to higher risk of diabetic complications, including stroke, and even higher risk of mortality. The findings from our previous work using data from another large healthcare system $^{22}$ indicated that both lower and higher $\mathrm{HbA1c}$ levels were associated with a higher risk of stroke, especially among women with type 2 diabetes. A large retrospective cohort including 246,544 adults with type 2 diabetes confirmed a U-shaped association between $\mathrm{HbA} 1 \mathrm{c}$ and cerebrovascular-related mortality. ${ }^{28}$ For macrovascular complications (the risk of stroke was not separately analyzed), patterns were almost linear for $\mathrm{HbA1c}$, with increased levels associated with higher risk of macrovascular complications. Another Swedish cohort ${ }^{27}$ including 271,174 patients with type 2 diabetes indicated that the U-shaped association for stroke risk with $\mathrm{HbA} 1 \mathrm{c}$ level was not apparent. They concluded that levels below the guideline target levels for $\mathrm{HbA} 1 \mathrm{c}$ and systolic blood pressure were associated with lower risks of stroke. These findings were partly consistent with the previous evidence from the Action to Control Cardiovascular Risk in Diabetes (ACCORD) trial ${ }^{29}$ that intensive glycemic control had a neutral effect on nonfatal cardiovascular events but increased cardiovascular-related death. Therefore, intensive glycemic control should be carefully considered in those patients with many cardiovascular risk factors and a relatively short lifespan. Our results also support the same suggestion that $\mathrm{HbA1c}$ level should be controlled properly within $6.0 \%$ to $7.0 \%$.

Very few studies have thus far focused on the association between $\mathrm{HbA} 1 \mathrm{c}$ level and hemorrhagic stroke among patients with diabetes (both type 1 and type 2). Data from the multi-ethnic Women's Health Initiative Observational Study ${ }^{30}$ showed that $\mathrm{HbA1c}$ was not predictive of hemorrhagic stroke $(H R, 0.86 ; 95 \%$ $\mathrm{Cl}, 0.32$ to 2.33). Another cohort from Finland ${ }^{31}$ did not report any data for the association between $\mathrm{HbA1c}$ level and hemor- 
rhagic stroke risk. However, we found a U-shaped association between $\mathrm{HbA1c}$ levels and risk of hemorrhagic stroke. In sensitivity analysis where stroke was diagnosed only by inpatient and emergency encounters, this U-shaped association was more pronounced. Although ischemic stroke and hemorrhagic stroke may not share the same pathophysiological mechanisms, patients with both poor glycemic control and intensive glycemic control ( $\mathrm{HbA1c}$ less than 6\%) were shown to be at greater risk of hemorrhagic stroke.

Poor glycemic control has been shown to be well correlated with macrovascular events in many studies and trials including the UKPDS ${ }^{32}$ and the Diabetes Control and Complications Trial (DCCT)/Epidemiology of Diabetes Interventions and Complications $(E D I C)^{33}$ programs. Results from $\mathrm{ACCORD}^{34}$, the Action in Diabetes and Vascular Disease: Preterax and Diamicron Modified Release Controlled Evaluation (ADVANCE), ${ }^{35}$ and Veteran's Affairs Diabetes Trial (VADT): ${ }^{36}$ however, did not support the fact that long-term intensive glycemic management can bring benefits to patients with type 2 diabetes. Despite the different study settings, our epidemiological analysis showed a consistent result with these trials, namely that both high and extremely low $\mathrm{HbA} 1 \mathrm{c}$ levels were associated with a high risk of both ischemic stroke and hemorrhagic stroke. Some underlying mechanism may help explain these findings. Lower $\mathrm{HbA1c}$ levels were always accompanied by more hypoglycemic events, more use of insulin, more use of combined therapies with other antidiabetic medications, and even more weight gain. These factors were, in general, associated with a high risk for comorbidities or mortalities. Notably, we also found that patients with lower $\mathrm{HbA} 1 \mathrm{c}$ levels were more likely to take antiplatelet or anticoagulant drugs. They also had a higher prevalence of atrial fibrillation. These findings may partly explain the higher risk in patients with lower $\mathrm{HbA1c}$ levels of both ischemic and hemorrhagic stroke. Other factors like pharmacological interactions, the different intensity of diabetes management and the conditions of patients themselves could also apply in explaining why lower HbA1c could contribute to a higher risk of stroke.

A major strength of this study was the large sample size, which allowed for high statistical power and the ability to perform stratified analyses. Further, the relatively rich clinical data and numerous events also make the results robust. The data we used was derived from administrative databases, avoiding the problem of differential recall bias. Data in this study were extracted from three partners of REACHnet, which minimizes the influence of low accessibility of health care. Inevitably, the study has several limitations. First, some socioeconomic variables were missing in the EMR data including education level, family in- come etc. Second, the stroke diagnoses in the present study were based on physician diagnosis and no chart review was performed. However, most American and European cohort studies, such as the Framingham Study, ${ }^{37}$ the Kaiser Permanente Medical Care Program ${ }_{1}^{38}$ and the Atherosclerosis Risk in Communities Study ${ }^{39}$ shared the same method used in our study to diagnose stroke events. Finally, our analyses adjusted for some confounding factors, however, unmeasured factors such as family history of diabetes, duration of diabetes, other related chronic diseases, dietary factors and physical activity status could not be evaluated. In addition, the relatively short follow-up duration may limit the generalizability of our findings.

\section{Conclusions}

The present study found a U-shaped association between $\mathrm{HbA} 1 \mathrm{c}$ levels and the risk of total, ischemic and hemorrhagic stroke among patients with type 2 diabetes, and the lowest risk was found among patients with $\mathrm{HbA} 1 \mathrm{c}$ at $6.0 \%$ to $7.0 \%$. Our findings supported the perspective that clinical diabetes management should be individualized according to the guideline recommendations rather than intensively seeking to lower $\mathrm{HbA} 1 \mathrm{c}$.

\section{Supplementary materials}

Supplementary materials related to this article can be found online at https://doi.org/10.5853/jos.2019.01704.

\section{Disclosure}

The authors have no financial conflicts of interest.

\section{Acknowledgments}

The LEAD study would like to acknowledge the contributions of our partners. The success of this study depended on their ongoing support and expertise. These partners include Ochsner Health System and the Ochsner Patient Research Advisory Board; Tulane Medical Center; University Medical Center New Orleans; REACHnet (a PCORnet CDRN) and their multi-stakeholder Diabetes Advisory Groups; Pennington Biomedical Research Center; Blue Cross and Blue Shield of Louisiana; and our patient and community partners Patricia Dominick, Catherine Glover, and Peggy Malone.

This work was supported by a Patient-Centered Outcomes Research (PCORI) cooperative agreement (NEN-1508-32257) as part of Natural Experiments for Translation in Diabetes 2.0 (NEXT-D2). All statements in this manuscript, including findings 
and conclusions, are solely those of the authors and do not necessarily represent the views of PCORI, its Board of Governors, or Methodology Committee. Drs. Shen, Hu, and Katzmarzyk were partly supported by a grant from the National Institute of General Medical Sciences (U54GM104940) of the National Institutes of Health.

\section{References}

1. Palmer MK, Toth PP. Trends in lipids, obesity, metabolic syndrome, and diabetes mellitus in the United States: an NHANES analysis (2003-2004 to 2013-2014). Obesity (Silver Spring) 2019;27:309-314.

2. Heron M. Deaths: leading causes for 2016. Natl Vital Stat Rep 2018;67:1-77.

3. Adler Al, Neil HA, Manley SE, Holman RR, Turner RC. Hyperglycemia and hyperinsulinemia at diagnosis of diabetes and their association with subsequent cardiovascular disease in the United Kingdom Prospective Diabetes Study (UKPDS 47). Am Heart J 1999;138:S353-S359.

4. Goto A, Noda M, Matsushita Y, Goto M, Kato M, Isogawa $A$, et al. Hemoglobin a1c levels and the risk of cardiovascular disease in people without known diabetes: a population-based cohort study in Japan. Medicine (Baltimore) 2015;94:e785.

5. Nichols GA, Joshua-Gotlib S, Parasuraman S. Glycemic control and risk of cardiovascular disease hospitalization and all-cause mortality. J Am Coll Cardiol 2013;62:121-127.

6. Shen Y, Shi L, Nauman E, Katzmarzyk PT, Price-Haywood EG, Bazzano AN, et al. Inverse association between HDL (highdensity lipoprotein) cholesterol and stroke risk among patients with type 2 diabetes mellitus. Stroke 2019;50:291297.

7. Shen Y, Shi L, Nauman E, Katzmarzyk PT, Price-Haywood EG, Yin $P$, et al. Race and sex differences in rates of diabetic complications. J Diabetes 2019;11:449-456.

8. Pathak RD, Schroeder EB, Seaquist ER, Zeng $C$, Lafata JE, Thomas $A$, et al. Severe hypoglycemia requiring medical intervention in a large cohort of adults with diabetes receiving care in U.S. integrated health care delivery systems: 20052011. Diabetes Care 2016;39:363-370.

9. Fleurence RL, Curtis LH, Califf RM, Platt R, Selby JV, Brown JS. Launching PCORnet, a national patient-centered clinical research network. J Am Med Inform Assoc 2014;21:578-582.

10. Wang Y, Katzmarzyk PT, Horswell R, Zhao W, Johnson J, Hu $G$. Comparison of the heart failure risk stratification performance of the CKD-EPI equation and the MDRD equation for estimated glomerular filtration rate in patients with type 2 diabetes. Diabet Med 2016;33:609-620.
11. Beckman JA, Creager MA, Libby P. Diabetes and atherosclerosis: epidemiology, pathophysiology, and management. JAMA 2002;287:2570-2581.

12. American Diabetes Association. 6. Glycemic targets: standards of medical care in diabetes-2018. Diabetes Care 2018;41:S55S64.

13. Skriver MV, Støvring H, Kristensen JK, Charles M, Sandbæk A. Short-term impact of HbA1c on morbidity and all-cause mortality in people with type 2 diabetes: a Danish population-based observational study. Diabetologia 2012;55:23612370.

14. Bots SH, van der Graaf Y, Nathoe HM, de Borst GJ, Kappelle $J \mathrm{~L}$, Visseren $\mathrm{FL}$, et al. The influence of baseline risk on the relation between $\mathrm{HbA} 1 \mathrm{c}$ and risk for new cardiovascular events and mortality in patients with type 2 diabetes and symptomatic cardiovascular disease. Cardiovasc Diabetol 2016;15:101.

15. Morita T, Okuno T, Himeno T, Watanabe K, Nakajima K, Koizumi $Y$, et al. Glycemic control and disability-free survival in hypoglycemic agent-treated community-dwelling older patients with type 2 diabetes mellitus. Geriatr Gerontol Int 2017;17:1858-1865.

16. Hwang JK, Lee SH, Song YB, Ahn J, Carriere K, Jang MJ, et al. Glycemic control status after percutaneous coronary intervention and long-term clinical outcomes in patients with type 2 diabetes mellitus. Circ Cardiovasc Interv 2017;10:e004157.

17. Heller SR, Bergenstal RM, White WB, Kupfer S, Bakris GL, Cushman WC, et al. Relationship of glycated haemoglobin and reported hypoglycaemia to cardiovascular outcomes in patients with type 2 diabetes and recent acute coronary syndrome events: the EXAMINE trial. Diabetes Obes Metab 2017;19:664-671.

18. Freemantle N, Danchin N, Calvi-Gries F, Vincent M, Home PD. Relationship of glycaemic control and hypoglycaemic episodes to 4-year cardiovascular outcomes in people with type 2 diabetes starting insulin. Diabetes Obes Metab 2016;18:152-158.

19. Fangel MV, Nielsen PB, Kristensen JK, Larsen TB, Overvad TF, Lip GYH, et al. Glycemic status and thromboembolic risk in patients with atrial fibrillation and type 2 diabetes mellitus. Circ Arrhythm Electrophysiol 2019;12:e007030.

20. Kranenburg G, van der Graaf $Y$, van der Leeuw J, Nathoe HM, de Borst GJ, Kappelle $\sqcup$, et al. The relation between $\mathrm{HbA1c}$ and cardiovascular events in patients with type 2 diabetes with and without vascular disease. Diabetes Care 2015;38:1930-1936.

21. Lin CC, Yang CP, Li Cl, Liu CS, Chen CC, Lin WY, et al. Visitto-visit variability of fasting plasma glucose as predictor of ischemic stroke: competing risk analysis in a national cohort of Taiwan Diabetes Study. BMC Med 2014;12:165.

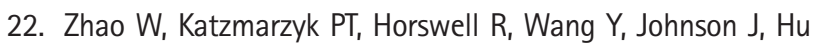


G. Sex differences in the risk of stroke and $\mathrm{HbA}(1 \mathrm{c})$ among diabetic patients. Diabetologia 2014;57:918-926.

23. Cederholm J, Zethelius B, Nilsson PM, Eeg-Olofsson K, Eliasson $B$, Gudbjörnsdottir $\mathrm{S}$, et al. Effect of tight control of $\mathrm{HbA} 1 \mathrm{c}$ and blood pressure on cardiovascular diseases in type 2 diabetes: an observational study from the Swedish National Diabetes Register (NDR). Diabetes Res Clin Pract 2009;86:74-81.

24. Camafort M, Alvarez-Rodríguez LR, Muñoz-Torrero JF, Sahuquillo JC, López-Jiménez $L$, Coll $R$, et al. Glucose control and outcome in patients with stable diabetes and previous coronary, cerebrovascular or peripheral artery disease. Findings from the FRENA Registry. Diabet Med 2011;28:73-80.

25. Hayashi T, Araki A, Kawashima S, Sone H, Watanabe H, Ohrui $\mathrm{T}$, et al. Metabolic predictors of ischemic heart disease and cerebrovascular attack in elderly diabetic individuals: difference in risk by age. Cardiovasc Diabetol 2013;12:10.

26. Alatorre $\mathrm{Cl}$, Hoogwerf BJ, Deeg MA, Nelson DR, Hunter TM, $\mathrm{Ng} \mathrm{WT}$, et al. Factors associated with stroke, myocardial infarction, ischemic heart disease, unstable angina, or mortality in patients from real world clinical practice with newlydiagnosed type 2 diabetes and early glycemic control. Curr Med Res Opin 2018;34:337-343.

27. Rawshani A, Rawshani A, Franzén S, Sattar N, Eliasson B, Svensson AM, et al. Risk factors, mortality, and cardiovascular outcomes in patients with type 2 diabetes. N Engl J Med 2018;379:633-644.

28. Kontopantelis E, Springate DA, Reeves D, Ashcroft DM, Rutter MK, Buchan I, et al. Glucose, blood pressure and cholesterol levels and their relationships to clinical outcomes in type 2 diabetes: a retrospective cohort study. Diabetologia 2015;58:505-518.

29. ACCORD Study Group. Nine-year effects of 3.7 years of intensive glycemic control on cardiovascular outcomes. Diabetes Care 2016;39:701-708.

30. Leening MJG, Cook NR, Franco OH, Manson JE, Lakshminara-

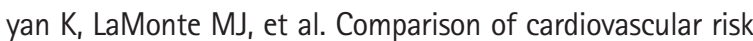

factors for coronary heart disease and stroke type in women. J Am Heart Assoc 2018;7:e007514.

31. Hägg $S$, Thorn LM, Forsblom CM, Gordin D, Saraheimo M, Tolonen $\mathrm{N}$, et al. Different risk factor profiles for ischemic and hemorrhagic stroke in type 1 diabetes mellitus. Stroke 2014:45:2558-2562.

32. Holman RR, Paul SK, Bethel MA, Matthews DR, Neil HA. 10Year follow-up of intensive glucose control in type 2 diabetes. N Engl J Med 2008;359:1577-1589.

33. Nathan DM, Cleary PA, Backlund JY, Genuth SM, Lachin JM, Orchard TJ, et al. Intensive diabetes treatment and cardiovascular disease in patients with type 1 diabetes. $N$ Engl J Med 2005;353:2643-2653.

34. Action to Control Cardiovascular Risk in Diabetes Study Group, Gerstein HC, Miller ME, Byington RP, Goff DC Jr, Bigger JT, et al. Effects of intensive glucose lowering in type 2 diabetes. N Engl J Med 2008;358:2545-2559.

35. ADVANCE Collaborative Group, Patel A, MacMahon S, Chalmers J, Neal B, Billot $L$, et al. Intensive blood glucose control and vascular outcomes in patients with type 2 diabetes. N Engl J Med 2008;358:2560-2572.

36. Duckworth W, Abraira C, Moritz T, Reda D, Emanuele N, Reaven $P D$, et al. Glucose control and vascular complications in veterans with type 2 diabetes. N Engl J Med 2009;360:129139.

37. Wilson PW, D'Agostino RB, Parise H, Sullivan L, Meigs JB. Metabolic syndrome as a precursor of cardiovascular disease and type 2 diabetes mellitus. Circulation 2005;112:3066-3072.

38. Kanaya AM, Adler N, Moffet HH, Liu J, Schillinger D, Adams $A$, et al. Heterogeneity of diabetes outcomes among asians and pacific islanders in the US: the diabetes study of Northern California (DISTANCE). Diabetes Care 2011;34:930-937.

39. Selvin E, Steffes MW, Zhu H, Matsushita K, Wagenknecht $L$, Pankow J, et al. Glycated hemoglobin, diabetes, and cardiovascular risk in nondiabetic adults. N Engl J Med 2010;362:800811. 
Supplementary Table 1. Risk of total stroke, ischemic stroke, and hemorrhagic stroke diagnosed only by inpatient or emergency encounters according to baseline and mean follow-up hemoglobin A1c level in patients with type 2 diabetes

\begin{tabular}{|c|c|c|c|c|c|c|c|}
\hline \multirow{2}{*}{ Variable } & \multicolumn{6}{|c|}{ Hemoglobin A1c (\%) } & \multirow{2}{*}{$P$ for trend } \\
\hline & $<6.0$ & $6.0-6.9$ & $7.0-7.9$ & $8.0-8.9$ & $9.0-9.9$ & $\geq 10$ & \\
\hline \multicolumn{8}{|l|}{ Baseline hemoglobin A1c level } \\
\hline No. of patients & 9,956 & 22,030 & 11,374 & 5,714 & 3,464 & 6,971 & \\
\hline \multicolumn{8}{|l|}{ Total stroke } \\
\hline No. of cases & 525 & 913 & 607 & 326 & 201 & 374 & \\
\hline Person-years & 35,729 & 88,528 & 47,601 & 23,299 & 13,748 & 26,117 & \\
\hline Age-and sex-adjustment & $1.40(1.26-1.56)$ & 1.00 & $1.32(1.19-1.47)$ & $1.62(1.42-1.84)$ & $1.89(1.62-2.21)$ & $2.24(1.98-2.54)$ & $<0.001$ \\
\hline Multivariable adjustment & $1.31(1.11-1.54)$ & 1.00 & $1.35(1.15-1.58)$ & $1.43(1.17-1.75)$ & $1.56(1.31-2.11)$ & $1.72(1.58-2.33)$ & $<0.001$ \\
\hline \multicolumn{8}{|l|}{ Ischemic stroke } \\
\hline No. of cases & 444 & 819 & 541 & 295 & 181 & 343 & \\
\hline Person-years & 35,592 & 88,330 & 47,460 & 23,229 & 13,702 & 26,063 & \\
\hline Age-and sex-adjustment & $1.31(1.17-1.47)$ & 1.00 & $1.32(1.18-1.47)$ & $1.63(1.43-1.86)$ & $1.90(1.61-2.23)$ & $2.28(2.00-2.59)$ & $<0.001$ \\
\hline Multivariable adjustment & $1.16(1.03-1.40)$ & 1.00 & $1.30(1.09-1.55)$ & $1.28(1.02-1.61)$ & $1.59(1.22-2.07)$ & $1.91(1.55-2.37)$ & $<0.001$ \\
\hline \multicolumn{8}{|l|}{ Hemorrhagic stroke } \\
\hline No. of cases & 81 & 94 & 66 & 31 & 20 & 31 & \\
\hline Person-years & 35,150 & 87,439 & 46,840 & 22,886 & 13,478 & 25,580 & \\
\hline Age- and sex-adjustment & $2.16(1.60-2.90)$ & 1.00 & $1.42(1.01-1.90)$ & $2.09(1.38-3.17)$ & $1.97(1.15-3.42)$ & $2.04(1.28-3.24)$ & $<0.001$ \\
\hline Multivariable adjustment & $1.93(1.37-2.72)$ & 1.00 & $1.59(1.10-2.30)$ & $2.21(1.44-3.40)$ & $2.04(1.16-3.58)$ & $1.89(1.16-3.08)$ & $<0.001$ \\
\hline \multicolumn{8}{|l|}{$\begin{array}{l}\text { Mean follow-up hemoglobin } \\
\text { A1c level }\end{array}$} \\
\hline No. of patients & 7,375 & 19,385 & 20,439 & 6,003 & 3,124 & 3,183 & \\
\hline \multicolumn{8}{|l|}{ Total stroke } \\
\hline No. of cases & 467 & 859 & 846 & 364 & 199 & 211 & \\
\hline Person-years & 28,156 & 79,213 & 77,190 & 25,094 & 12,848 & 12,520 & \\
\hline Age- and sex-adjustment & $1.46(1.31-1.64)$ & 1.00 & $1.10(1.00-1.21)$ & $1.68(1.49-1.90)$ & $2.12(1.82-2.48)$ & $2.69(2.31-3.14)$ & $<0.001$ \\
\hline Multivariable adjustment & $1.40(1.17-1.67)$ & 1.00 & $1.19(1.03-1.37)$ & $1.45(1.19-1.76)$ & $1.95(1.54-2.48)$ & $2.55(2.03-3.21)$ & $<0.001$ \\
\hline \multicolumn{8}{|l|}{ Ischemic stroke } \\
\hline No. of cases & 391 & 773 & 751 & 335 & 181 & 192 & \\
\hline Person-years & 28,016 & 79,026 & 77,014 & 25,034 & 12,807 & 12,479 & \\
\hline Age- and sex-adjustment & $1.36(1.20-1.53)$ & 1.00 & $1.08(0.98-1.19)$ & $1.72(1.51-1.96)$ & $2.14(1.82-2.53)$ & $2.72(2.31-3.19)$ & $<0.001$ \\
\hline Multivariable adjustment & $1.19(1.03-1.46)$ & 1.00 & $1.13(0.96-1.33)$ & $1.40(1.13-1.74)$ & $1.96(1.51-2.54)$ & $2.34(1.81-3.01)$ & $<0.001$ \\
\hline \multicolumn{8}{|l|}{ Hemorrhagic stroke } \\
\hline No. of cases & 76 & 86 & 95 & 29 & 18 & 19 & \\
\hline Person-years & 27,634 & 78,152 & 76,207 & 24,594 & 12,565 & 12,220 & \\
\hline Age- and sex-adjustment & $2.41(1.77-3.29)$ & 1.00 & $1.59(1.14-2.22)$ & $1.65(1.03-2.64)$ & $1.86(1.01-3.48)$ & $2.67(1.67-4.18)$ & $<0.001$ \\
\hline Multivariable adjustment & $2.32(1.61-3.35)$ & 1.00 & $1.48(1.05-2.07)$ & $1.66(1.03-2.68)$ & $1.82(0.96-3.43)$ & $2.30(1.21-3.22)$ & $<0.001$ \\
\hline
\end{tabular}

Values are presented as hazard ratio (95\% confidence interval) unless otherwise indicated. Multivariable adjustment included age, sex, race, body mass index, systolic blood pressure, low-density lipoprotein cholesterol, high-density lipoprotein cholesterol, triglycerides, estimated glomerular filtration rate, smoking, insurance type, use of antihypertensive drugs, use of glucose lowering drugs, use of lipid-lowering drugs, use of antiplatelet or anticoagulant drugs, and presence of atrial fibrillation. 
Supplementary Table 2. Subgroup analyses for the risk of total stroke by baseline hemoglobin A1c in patients excluding those who died during the follow-up

\begin{tabular}{|c|c|c|c|c|c|c|c|}
\hline \multirow{2}{*}{ Variable } & \multicolumn{6}{|c|}{ Hemoglobin A1c (\%) } & \multirow{2}{*}{$P$ for trend } \\
\hline & $<6.0$ & $6.0-6.9$ & 7.0-7.9 & $8.0-8.9$ & $9.0-9.9$ & $\geq 10$ & \\
\hline \multicolumn{8}{|l|}{ Baseline hemoglobin A1c level } \\
\hline No. of patients & 10,648 & 24,385 & 12,522 & 6,292 & 3,701 & 7,392 & \\
\hline No. of cases & 1,131 & 2,501 & 1,478 & 762 & 419 & 755 & \\
\hline Person-years & 38,309 & 95,042 & 50,694 & 24,838 & 14,337 & 27,169 & \\
\hline Age-and sex-adjustment & $1.10(1.03-1.18)$ & 1.00 & $1.18(1.11-1.26)$ & $1.35(1.25-1.47)$ & $1.44(1.30-1.60)$ & $1.59(1.47-1.73)$ & $<0.001$ \\
\hline Multivariable adjustment & $1.08(1.01-1.11)$ & 1.00 & $1.13(1.05-1.20)$ & $1.22(1.12-1.33)$ & $1.25(1.13-1.39)$ & $1.36(1.24-1.38)$ & $<0.001$ \\
\hline \multicolumn{8}{|l|}{$\begin{array}{l}\text { Mean follow-up hemoglobin } \\
\text { A1c level }\end{array}$} \\
\hline No. of patients & 8,153 & 21,580 & 21,841 & 6,595 & 3,400 & 3,371 & \\
\hline No. of cases & 1,021 & 2,269 & 2,155 & 800 & 410 & 391 & \\
\hline Person-years & 30,428 & 85,219 & 81,526 & 26,676 & 13,579 & 12,959 & \\
\hline Age-and sex-adjustment & $1.21(1.12-1.30)$ & 1.00 & $1.07(1.01-1.13)$ & $1.38(1.27-1.50)$ & $1.61(1.45-1.79)$ & $1.85(1.65-2.06)$ & $<0.001$ \\
\hline Multivariable adjustment & $1.14(1.06-1.24)$ & 1.00 & $1.05(1.00-1.11)$ & $1.21(1.11-1.32)$ & $1.40(1.26-1.56)$ & $1.51(1.35-1.69)$ & $<0.001$ \\
\hline
\end{tabular}

Values are presented as hazard ratio (95\% confidence interval) unless otherwise indicated. Multivariable adjustment included age, sex, race, body mass index, systolic blood pressure, low-density lipoprotein cholesterol, high-density lipoprotein cholesterol, triglycerides, estimated glomerular filtration rate, smoking, insurance type, use of antihypertensive drugs, use of glucose lowering drugs, use of lipid-lowering drugs, use of antiplatelet or anticoagulant drugs, and presence of atrial fibrillation. 
Supplementary Table 3. Subgroup analyses for the risk of total stroke diagnosed only by inpatient and emergency according to baseline hemoglobin A1c levels in patients with type 2 diabetes

\begin{tabular}{|c|c|c|c|c|c|c|c|c|}
\hline \multirow{2}{*}{ Variable } & \multirow{2}{*}{$\begin{array}{l}\text { No. of } \\
\text { patients }\end{array}$} & \multicolumn{6}{|c|}{ Hemoglobin A1c (\%) } & \multirow{2}{*}{$\begin{array}{l}P \text { for } \\
\text { trend }\end{array}$} \\
\hline & & $<6.0$ & $6.0-6.9$ & $7.0-7.9$ & $8.0-8.9$ & $9.0-9.9$ & $\geq 10$ & \\
\hline \multicolumn{9}{|l|}{ Age (yr) } \\
\hline$<60$ & 19,414 & $1.23(0.78-1.93)$ & 1.00 & $1.09(0.54-1.46)$ & $1.23(0.73-2.06)$ & $1.81(1.11-2.96)$ & $1.55(1.02-2.34)$ & 0.033 \\
\hline$\geq 60$ & 40,095 & $1.25(1.05-1.49)$ & 1.00 & $1.39(1.18-1.65)$ & $1.40(1.13-1.74)$ & $1.42(1.08-1.88)$ & $1.74(1.40-2.17)$ & $<0.001$ \\
\hline \multicolumn{9}{|l|}{ Sex } \\
\hline Man & 28,239 & $1.20(1.01-1.53)$ & 1.00 & $1.39(1.10-1.76)$ & $1.38(1.03-1.85)$ & $1.30(0.89-1.91)$ & $1.87(1.39-2.51)$ & 0.001 \\
\hline Women & 31,270 & $1.39(1.11-1.73)$ & 1.00 & $1.31(1.05-1.63)$ & $1.47(1.12-1.93)$ & 1.99 (1.46-2.70) & $1.92(1.48-2.50)$ & $<0.001$ \\
\hline \multicolumn{9}{|l|}{ Race } \\
\hline African Americans & 24,140 & $1.31(1.01-1.72)$ & 1.00 & $1.18(0.91-1.53)$ & $1.36(1.00-1.85)$ & $1.74(1.24-2.45)$ & $1.79(1.35-2.37)$ & $<0.001$ \\
\hline Whites & 35,369 & $1.30(1.06-1.60)$ & 1.00 & $1.47(1.20-1.79)$ & $1.49(1.15-1.94)$ & $1.56(1.11-2.19)$ & $2.00(1.52-2.64)$ & $<0.001$ \\
\hline \multicolumn{9}{|l|}{ Body mass index $\left(\mathrm{kg} / \mathrm{m}^{2}\right)$} \\
\hline$<30$ & 22,847 & $1.29(1.05-1.59)$ & 1.00 & $1.38(1.11-1.72)$ & $1.65(1.26-2.16)$ & $1.67(1.18-2.35)$ & $1.85(1.39-2.46)$ & $<0.001$ \\
\hline$\geq 30$ & 36,662 & $1.40(1.08-1.82)$ & 1.00 & $1.29(1.02-1.63)$ & $1.18(0.88-1.59)$ & $1.62(1.16-2.25)$ & $1.93(1.47-2.53)$ & $<0.001$ \\
\hline \multicolumn{9}{|l|}{ Smoking status } \\
\hline Never smoking & 47,965 & $1.27(1.05-1.54)$ & 1.00 & $1.50(1.26-1.80)$ & $1.61(1.29-2.01)$ & $1.77(1.35-2.33)$ & $1.94(1.56-2.43)$ & $<0.001$ \\
\hline Past and current smoking & 11,544 & $1.41(1.02-1.94)$ & 1.00 & $1.02(0.65-1.34)$ & $1.26(0.61-1.55)$ & $1.47(0.88-2.45)$ & $1.89(1.25-2.86)$ & 0.012 \\
\hline \multicolumn{9}{|l|}{$\begin{array}{l}\text { Using antidiabetic } \\
\text { medications* }\end{array}$} \\
\hline No & 16,393 & $1.06(0.82-1.36)$ & 1.00 & $1.33(0.94-1.88)$ & $1.43(0.86-2.36)$ & $1.63(0.85-3.13)$ & $1.07(0.84-2.02)$ & 0.246 \\
\hline Yes & 43,116 & $1.54(1.24-1.91)$ & 1.00 & $1.39(1.16-1.67)$ & $1.47(1.18-1.84)$ & $1.71(1.32-2.21)$ & $2.01(1.63-2.48)$ & $<0.001$ \\
\hline Insulin or sulfonylurea & 17,691 & $1.44(1.12-1.86)$ & 1.00 & $1.12(0.91-1.38)$ & $1.16(1.02-1.48)$ & $1.33(1.01-1.75)$ & $1.49(1.18-1.87)$ & 0.013 \\
\hline Others & 25,425 & $1.28(1.03-1.58)$ & 1.00 & $1.38(1.06-1.79)$ & $1.38(0.91-2.09)$ & $1.43(0.79-2.59)$ & $1.12(0.60-2.08)$ & 0.114 \\
\hline \multicolumn{9}{|l|}{$\begin{array}{l}\text { Using lipid-lowering } \\
\text { medications* }\end{array}$} \\
\hline No & 24,027 & $1.31(1.01-1.73)$ & 1.00 & $1.37(1.02-1.83)$ & $1.26(1.01-1.85)$ & $2.01(1.34-3.02)$ & $1.73(0.94-3.24)$ & 0.013 \\
\hline Yes & 35,482 & $1.30(1.06-1.60)$ & 1.00 & $1.38(1.14-1.68)$ & $1.56(1.23-1.98)$ & $1.57(1.17-2.11)$ & $2.27(1.80-2.85)$ & $<0.001$ \\
\hline \multicolumn{9}{|l|}{$\begin{array}{l}\text { Using antihypertensive } \\
\text { medications* }\end{array}$} \\
\hline No & 14,069 & $2.00(1.22-3.29)$ & 1.00 & $1.88(1.13-3.11)$ & $2.06(1.10-3.86)$ & $2.53(1.25-5.10)$ & $2.25(1.21-4.17)$ & 0.021 \\
\hline Yes & 45,440 & $1.24(1.04-1.48)$ & 1.00 & $1.30(1.10-1.54)$ & $1.38(1.12-1.71)$ & $1.60(1.24-2.06)$ & $1.90(1.54-2.33)$ & $<0.001$ \\
\hline \multicolumn{9}{|l|}{$\begin{array}{l}\text { Using antiplatelet or } \\
\text { anticoagulant drugs* }\end{array}$} \\
\hline No & 47,384 & $1.50(1.17-1.93)$ & 1.00 & $1.54(1.22-1.94)$ & $1.82(1.37-2.42)$ & $2.40(1.71-3.37)$ & $2.55(1.91-3.41)$ & $<0.001$ \\
\hline Yes & 12,125 & $1.19(0.96-1.49)$ & 1.00 & $1.18(0.95-1.47)$ & $1.14(0.86-1.51)$ & $1.17(0.84-1.64)$ & $1.48(1.13-1.92)$ & 0.072 \\
\hline \multicolumn{9}{|l|}{$\begin{array}{l}\text { Presence of atrial } \\
\text { fibrillation* }\end{array}$} \\
\hline No & 52,065 & $1.45(1.17-1.81)$ & 1.00 & $1.42(1.17-1.74)$ & $1.51(1.18-1.93)$ & $1.98(1.51-2.59)$ & $1.91(1.51-2.41)$ & $<0.001$ \\
\hline Yes & 7,444 & $1.11(0.87-1.44)$ & 1.00 & $1.25(0.96-1.64)$ & $1.28(0.90-1.81)$ & $1.30(0.88-1.57)$ & $2.01(1.40-2.87)$ & 0.002 \\
\hline
\end{tabular}

Values are presented as hazard ratio (95\% confidence interval) unless otherwise indicated. All hazard ratios were adjusted for age, sex, race, body mass index, systolic blood pressure, low-density lipoprotein cholesterol, high-density lipoprotein cholesterol, triglycerides, estimated glomerular filtration rate, smoking, insurance type, use of antihypertensive drugs, use of glucose lowering drugs, use of lipid-lowering drugs, use of antiplatelet or anticoagulant drugs, and presence of atrial fibrillation other than the variable for stratification.

*P for interaction $<0.05$. 